\title{
MASS TO LIGHT RATIOS OF LARGE MAGELLANIC CLOUD CLUSTERS
}

\author{
MARIO MATEO \\ The Observatories of the Carnegie Institution of Washington \\ 813 Santa Barbara Street \\ Pasadena, CA 91101, USA \\ DOUGLAS WELCH, PHIL FISCHER \\ Dept. of Physics \\ McMaster University \\ Hamilton, ON L8S 4M1, Canada
}

\begin{abstract}
The massive star clusters of the Large Magellanic Cloud (LMC) are ideal laboratories for the study of the dynamical evolution of rich stellar systems. Not only do they contain large numbers of stars but, unlike the Galactic globular clusters, LMC clusters span a large range in age. Here we describe a continuing project to determine the temporal evolution of the mass-to-light $(\mathrm{M} / \mathrm{L})$ ratio of LMC clusters and present some preliminary results.
\end{abstract}

The crucial observational input required for the determination of the M/L ratio of a cluster is its central velocity dispersion. With the photon-counting échelle spectrograph at Las Campanas Observatory, we used two methods to measure this parameter. First, we obtained velocities of individual cluster red giants with a typical precision of about $1-2 \mathrm{~km} \mathrm{~s}^{-1}$ per observation to $V \sim$ 18.5. The results for three clusters observed in this manner are summarized in the first three lines of Table 1. The third column of the table lists the number of individual stars observed in each cluster. Second, we obtained spectra of the integrated light of the cores of five old LMC clusters and used cross-correlation techniques to estimate their internal velocity dispersions from the observed line-broadening. These spectra were obtained using a 1" $\mathrm{x} 4$ " slit centred on the compact cores of these clusters. The resulting central velocity dispersons and their uncertainties are listed in the Table. We note that our velocity dispersion for NGC 1835 agrees well with the value obtained recently by Dubath et al. (1990) using a similar technique.

Table 1. LMC Cluster Mass-to-Light Ratios.

\begin{tabular}{ccccccr}
\hline \hline Cluster & $\begin{array}{c}\sigma_{v} \\
\mathrm{~km} / \mathrm{s}\end{array}$ & $N$ & $\begin{array}{c}\Sigma_{0, V} \\
\mathrm{mag}\end{array}$ & $\begin{array}{c}r_{c} \\
\mathrm{pc}\end{array}$ & $\begin{array}{c}\log (\text { Age }) \\
\text { yrs }\end{array}$ & $\log (M / L)$ \\
\hline NGC 1783 & $4.7 \pm 0.5$ & 54 & 18.5 & 2.9 & 9.2 & $0.23 \pm 0.15$ \\
NGC 2157 & $2.8 \pm 0.6$ & 28 & 16.5 & 2.4 & 7.9 & $-0.96 \pm 0.17$ \\
NGC 2257 & $5.5 \pm 1.0$ & 35 & 20.2 & 4.6 & 10.2 & $0.81 \pm 0.19$ \\
NGC 1786 & $9.9 \pm 3.0$ & $\ldots$ & 16.1 & 0.7 & 10.2 & $0.53 \pm 0.30$ \\
NGC 1835 & $11.5 \pm 2.5$ & $\ldots$ & 15.5 & 0.8 & 10.2 & $0.36 \pm 0.28$ \\
NGC 1916 & $16.6 \pm 4.0$ & $\ldots$ & 15.8 & 0.5 & 10.2 & $1.03 \pm 0.36$ \\
NGC 2005 & $15.3 \pm 4.3$ & $\ldots$ & 15.8 & $<0.5$ & 10.2 & $0.96 \pm 0.30$ \\
NGC 2019 & $11.7 \pm 3.0$ & $\ldots$ & 15.5 & $<0.5$ & 10.2 & $0.58 \pm 0.30$ \\
\hline
\end{tabular}

191

R. Haynes and D. Milne (eds.), The Magellanic Clouds, 191-192.

(C) 1991 IAU. Printed in the Netherlands. 
To determine the $\mathrm{M} / \mathrm{L}$ ratios of the program clusters we performed CCD surface photometry or drew the necessary data from the literature. The resulting $\mathrm{M} / \mathrm{L}$ ratios are listed in Table 1 , and were determined using the method described by Armandroff and Da Costa (1986) and Peterson and Latham (1986). Ages for the clusters were estimated from our own colour-magnitude diagrams of these systems or from results in the literature. The five clusters for which we have obtained integrated spectra are so compact and lie in such crowded fields that we have no reliable independent age estimates for them. We have arbitrarily assumed that they are as old as NGC 2257.

The behaviour of $\mathrm{M} / \mathrm{L}$ ratio as a function of age is shown in Fig. 1, along with the prediction of the model of Elson et al. (1987) for three different IMF slopes. The model results have been shifted downward by 0.3 in $\log (M / L)$ to agree with the range of $\mathrm{M} / \mathrm{L}$ ratios observed in globular clusters by Illingworth (1976) which are schematically illustrated as a dashed box. Our observations of LMC clusters appear to be most consistent with the evolutionary curve based on an IMF slope that is somewhat steeper than the Salpeter value. Fig. 1 also shows that the mean $M / L$ ratios of some of the oldest LMC clusters lie outside the range exhibited by Galactic globular clusters. The large uncertainties in the velocity dispersions derived from the integrated spectra make this result tentative. However, the M/L ratio derived from NGC 2257 from observations of individual stars is also higher than the range observed in Galactic globulars. These results suggest that the oldest LMC and Galaxy clusters may be dynamically distinct.

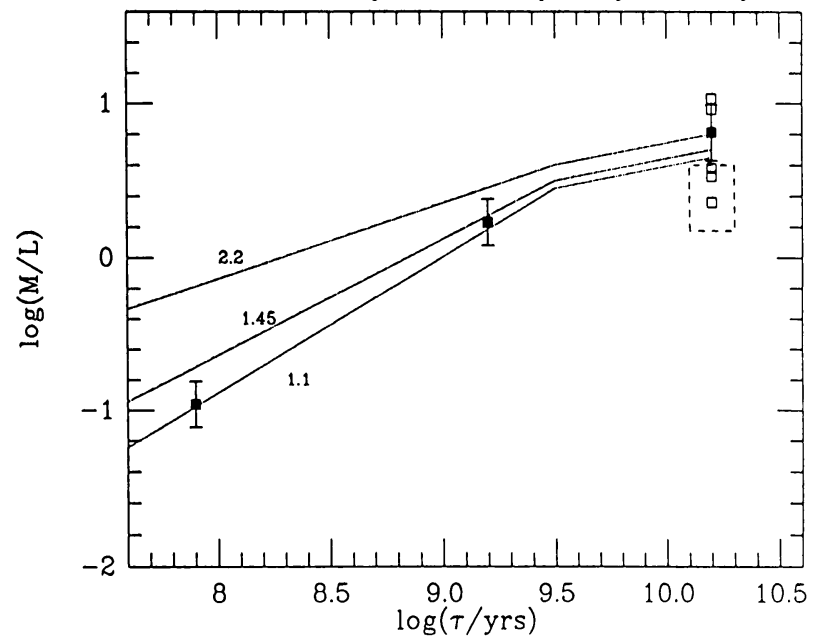

Figure 1. M/L (in solar units)v. age for the clusters listed in Table 1. The closed squares denote the three clusters for which the velocity dispersions were obtained from measurements of individual members. The open squares represent the clusters for which integrated spectra were obtained. Remaining symbols are described in the text.

\section{References}

Armandroff, T.E., Da Costa, G.S. (1986), A.J. 92, 777.

Dubath, P., Meylan, G., Mayor, M. (1990), Astr. Ap. in press.

Elson, R.A.W., Fall, S.M., Freeman, K.C. (1987), Ap. J. 323, 54.

Illingworth, G. (1976), Ap. J. 204, 73.

Peterson, R.C., Latham, D.W. (1986), Ap. J. 305, 645. 